\title{
Evaluación de un medio para la conservación de Streptococcus pneumoniae a partir de muestras nasofaríngeas
}

\author{
Nacxiry Fonseca, Maria Claudia Vela, Elizabeth Castañeda \\ Grupo de Microbiologia, Instituto Nacional de Salud, Bogotá, D.C., Colombia
}

\begin{abstract}
La Organización Mundial de la Salud (OMS) llegó a un consenso, en 1998, acerca de la metodología general para el estudio de portadores de Streptococcus pneumoniae. Esta metodología incluía un nuevo medio para el transporte y el almacenamiento de muestras nasofaríngeas, compuesto por triptona, glucosa, glicerol y leche descremada, denominado STGG. Este medio fue evaluado por nuestro laboratorio, en marzo de 1999, en un grupo de 15 niños menores de 5 años asistentes a una guardería de Bogotá. Se les tomó una muestra nasofaríngea que se colocó en el medio STGG y, posteriormente, se valoró el crecimiento de S. pneumoniae en cuatro momentos diferentes: $0,5,10$ y 30 días después de la toma de la muestra. La recuperación inicial de S. pneumoniae fue de 73\% (11/15). En todas las 11 muestras de las que se obtuvo un crecimiento inicial, fue posible recuperar $S$. pneumoniae en los diferentes tiempos de observación, aún en las muestras con crecimiento en un solo cuadrante. En el tiempo 0 y a los 30 días, 7 de las 11 muestras presentaron un crecimiento de abundante a moderado. Con los datos obtenidos, se logró demostrar que el medio STGG es capaz de mantener, a largo plazo, la viabilidad de $S$. pneumoniae en muestras nasofaríngeas de niños portadores de este microorganismo.
\end{abstract}

Palabras clave: Streptococcus pneumoniae, medio STGG, conservación, muestras nasofaríngeas.

\section{Evaluation of a medium for the conservation of Streptococcus pneumoniae in nasopharyngeal isolates}

The World Health Organization (WHO) reached a consensus on a core methodology for pneumococcal carrier studies in 1998. This methodology included a new medium to transport and to store nasopharyngeal samples, called skim milk-tryptone-glucose-glycerin (STGG). This medium was evaluated by our laboratory in March 1999 in a group of 15 children less than 5 years old attending a daycare center in Santa Fe de Bogotá. The nasopharyngeal samples were placed in the STGG medium and, subsequently, S. pneumoniae growth was semiquantified at 4 different times: $0,5,10$ and 30 days. The initial recovery of $S$. pneumoniae was $73 \%(11 / 15)$. In the 11 samples with initial growth it was possible to isolate $S$. pneumoniae in all the different times of observation, even in samples with individual colonies. At time 0 and 30 days after, 7 of 11 samples showed moderate and abundant growth. The data obtained in this study proved that STGG medium has the ability to maintain the $S$. pneumoniae viable over time from nasopharyngeal samples collected from children carriers.

Key words: Streptococcus fneumoniae, STGG medium, conservation, nasopharyngeal isolates.

Streptococcus pneumoniae es una causa importante de morbilidad y mortalidad a escala mundial (1). La infección neumocócica está

\section{Correspondencia:}

M. C. Vela, Grupo de Microbiologia, Instituto Nacional de Salud, Apartado aéreo 80080, Bogotá, D.C., Colombia mvela@hemagogus.ins,gov.co

Recibido: 13/06/00; aceptado: 04/08/00 usualmente precedida por la colonización de la nasofaringe humana, siendo ésta un importante factor de riesgo para desarrollar la enfermedad $(2,3)$. En un estudio realizado en 158 niños de Papua, Nueva Guinea, se demostró que más de $95 \%$ de ellos eran colonizados durante sus primeros dos años de vida por S. pneumoniae (4); otros estudios han señalado que los niños 
colonizados desarrollan con más frecuencia otitis media aguda, que los que no lo están (5-7).

En 1998, la Organización Mundial de la Salud (OMS) llegó a un consenso acerca de una metodología para el estudio de portadores de $S$. pneumoniae, la cual emplea un nuevo medio compuesto por triptona, glucosa, glicerol y leche descremada (STGG). Este medio podría ser empleado en un sistema de vigilancia con muestras nasofaríngeas, lo que permitiría determinar, con un método no invasor, tanto la circulación de los serotipos de S. pneumoniae como su patrón de susceptibilidad. Esto, a su vez, permitiría correlacionar los tipos capsulares que colonizan la nasofaringe con los causantes de enfermedad invasora.

El objetivo de este trabajo fue el de evaluar la habilidad del medio STGG para mantener la viabilidad de $S$. pneumoniae, con el propósito de ser implementado, en el futuro, en un sistema de vigilancia.

\section{Materiales y métodos}

Población: se estudiaron 15 niños menores de 5 años asistentes a un jardín hogar infantil del Instituto Colombiano de Bienestar Familiar de Bogotá. El protocolo contó con la aprobación de la directora de la institución.

Toma de la muestra: la muestra nasofaríngea se tomó con un escobillón pediátrico de alginato de calcio con mango de aluminio flexible (Fisher Brand), el cual se introdujo por la fosa nasal y se dejó en la nasofarínge por 5 segundos para saturar toda la punta del escobillón.

Medio STGG: el medio se preparó con $3 \mathrm{~g} / \mathrm{ml}$ de caldo triptona-soya (BBL), $0,5 \mathrm{~g} / \mathrm{ml}$ de glucosa (Sigma), $2 \mathrm{~g} / \mathrm{ml}$ de leche descremada en polvo (Difco), $10 \mathrm{ml}$ de glicerol (Sigma) y $100 \mathrm{ml}$ de agua bidestilada; se dispensó en alícuotas de $1 \mathrm{ml}$ en criovales (Nalgene) y se esterilizó en autoclave a $15 \mathrm{lb}$ durante 10 minutos.

Procesamiento de las muestras: el escobillón con la muestra nasofaríngea se colocó inmediatamente en un vial con el medio STGG. Una hora después, en el laboratorio, se agitó el vial en vórtex de 10 a 20 segundos para desprender los microorganismos del escobillón; se realizó una siembra para la recuperación inicial de los microorganismos y se colocó el vial con el escobillón a $-70^{\circ} \mathrm{C}$.

Para la valoración inicial de los microorganismos en la muestra (día 0), se inocularon $50 \mathrm{ml}$ en agar sangre de cordero al $5 \%$ con gentamicina $(5 \mu \mathrm{g} /$ $\mathrm{ml}$, Sigma) y se sembró por agotamiento. Los cultivos se incubaron a $37^{\circ} \mathrm{C}$ en $5 \%$ de $\mathrm{CO}_{2}$ durante 18 horas.

Para evaluar la preservación de $\mathbf{S}$. pneumoniae en los diferentes tiempos (5, 10 y 30 días), los viales se retiraron del congelador, se descongelaron, se obtuvieron las muestras y se volvieron a congelar. Las muestras se procesaron en la forma descrita anteriormente.

Semicuantificación: posterior a la incubación, se observó la morfología macroscópica y se valoró el crecimiento, teniendo en cuenta el número de cuadrantes y, a su vez, el número de colonias en cada uno de los cuadrantes, de la siguiente manera: abundante, crecimiento en 4 cuadrantes $(++++)$; moderado, crecimiento en 3 cuadrantes $(+++)$; leve, crecimiento en 2 cuadrantes $(++)$, y colonias individuales, crecimiento en un solo cuadrante $(+)$.

Identificación: las colonias que presentaron morfología compatible con S. pneumoniae por tamaño y alfa-hemólisis se resembraron en agar sangre de caballo al $10 \%$. A partir de este cultivo se realizó la identificación con base en técnicas previamente estandarizadas, como son la coloración de Gram, la prueba de susceptibilidad a la optoquina $(5 \mathrm{mg}$ ) con discos de $10 \mathrm{~mm}$ (Difco) y la solubilidad en bilis (8), con el empleo del desoxicolato de sodio al 10\% (Sigma). Los tipos capsulares se establecieron con la reacción de Quellung, empleando 12 mezclas de antisueros del Statens Seruminstitut de Copenhague, Dinamarca (9).

\section{Resultados}

S. pneumoniae fue aislado inicialmente (día 0) en $73 \%(11 / 15)$ de las muestras. La recuperación de la bacteria a partir del medio STGG en los diferentes tiempos estudiados se presenta en el cuadro 1. En todas las muestras en las que se 
Cuadro 1. Evaluación del crecimiento de Streptococcus pneumoniae a partir del medio STGG en los diferentes tiempos estudiados.

\begin{tabular}{ccccc}
\hline & \multicolumn{4}{c}{ Tiempo de observación (dias) } \\
\cline { 2 - 5 } Muestra & 0 & 5 & 10 & 30 \\
\hline 1 & +++ & ++ & ++ & +++ \\
2 & +++ & ++ & ++ & +++ \\
3 & +++ & + & ++ & +++ \\
4 & +++ & + & + & ++ \\
5 & + & + & + & + \\
6 & + & + & + & + \\
7 & ++++ & ++ & +++ & +++ \\
8 & +++ & + & ++ & +++ \\
9 & ++ & ++ & ++ & +++ \\
10 & +++ & ++ & ++ & +++ \\
11 & + & + & + & + \\
\hline
\end{tabular}

++++ abundante; +++ moderado; ++ leve; + colonias individuales.

obtuvo un crecimiento inicial fue posible recuperar S. pneumoniae en los diferentes tiempos de observación, aún en aquéllas en las que el crecimiento inicial fue de colonias individuales en un solo cuadrante. En el tiempo cero y a los 30 días, en 7 de las 11 muestras se obtuvo un crecimiento abundante o moderado. Igualmente, a los 30 días, 4 muestras presentaron el mismo crecimiento del tiempo cero y 3 conservaron el mismo número de colonias individuales a lo largo del período de observación.

Macroscópicamente, se observaron colonias de tipo mucoide en 4 muestras y colonias de tipo liso en 3. También se observó que 3 aislamientos tenían 2 tipos de colonias (mucoide y lisa) en el día 0 y perdieron un tipo en los otros tiempos evaluados; el tipo de colonia que permaneció fue el más abundante en la muestra inicial; sin embargo, en una muestra con 2 tipos de colonias, lisa grande y pequeña, los 2 se recuperaron en todos los tiempos evaluados.

Para confirmar la morfología macroscópica, se determinó el tipo capsular en 8 de los 11 aislamientos; predominó el serotipo 3 con $62,5 \%$ (5/8). Otros serotipos encontrados fueron 4, 19F, 18 y no serotipificable.

\section{Discusión}

Los resultados obtenidos demuestran que el medio STGG es un medio útil, tanto para el transporte de muestras nasofaríngeas como para la conservación de la bacteria en el mismo, dado que todos los aislamientos permanecieron viables en él hasta por 30 días, sin importar las descongelaciones realizadas en los diferentes tiempos evaluados. Esta experiencia confirma lo informado en dos estudios de colonización nasofaríngea por $S$. pneumoniae realizados en Chile, en los que emplearon con éxito este medio $(12,13)$ para la conservación de las muestras.

Es importante anotar que en algunas muestras se recuperaron inicialmente dos tipos de colonias y una de ellas, la que estaba en menor cantidad, no se pudo recuperar posteriormente. Esta observación cualitativa debe confirmarse con un estudio en el que se emplee un número mayor de muestras.

El medio es fácil de preparar y sus componentes se adquieren también con facilidad; permite la inoculación de varias cajas de agar sangre a partir de la muestra nasofaríngea original, así como cuantificar el crecimiento de los organismos. Entre las desventajas se podría señalar que no se encuentra disponible en el mercado y el control de calidad no se ha establecido aún.

Adicionalmente, la toma de la muestra nasofaríngea no requiere un procedimiento invasor para su obtención y al confirmarse la utilidad del medio se podría pensar en un estudio de vigilancia de serotipos y de susceptibilidad antimicrobiana con portadores. Es importante señalar que una de las fallas en la toma de una buena muestra nasofaríngea puede deberse a que el escobillón no alcanza a llegar a la nasofarínge, ya que los niños sienten obstrucción e incomodidad, impidiendo la penetración completa del mismo.

Este medio se puede emplear en los centros hospitalario, dado que la conservación de la muestra permite que se realicen estudios que permitan correlacionar el tipo capsular causante de enfermedad invasora con los tipos capsulares que colonizan la nasofaringe.

\section{Referencias}

1. Centers for Disease Control and Prevention. Defining the public health impact of drug-resistant Streptococcus pneumoniae: report of a working group. MMWR 1996; 45:1-16. 
2. World Health Organization, Programme for the Control of Acute Respiratory Infections. Pneumococcal conjugate vaccines. Report of a meeting. Geneva: WHO, 15-17 November, 1993. p.1-18.

3. Dejsirilert S, Overweg K, Sluijter M, Saengsuk L, Gratten M, Ezaki T, et al. Nasopharyngeal carriage of penicillin-resistant Streptococcus pneumoniae among children with acute respiratory tract infections in Thailand: a molecular epidemiological survey. J Clin Microbiol 1999; $37: 1832-8$.

4. Montgomery JM, Lehman D, Smith T, Michael A, Joseph B, Lupiwa $T$, et al. Bacterial colonization of the upper respiratory tract and its association with acute lower respiratory tract infections in Highland children of Papua, New Guinea. Rev Infect Dis 1990;12(suppl 8):\$1006-16.

5. Sluijter M, Faden H, De Groot R, Lemmens $\mathbf{N}$, Goessens WHF, Van Belkum A, et al. Molecular characterization of pneumococcal nasopharynx isolates collected from children during their first 2 years of life. $\mathrm{J}$ Clin Microbiol 1998;36:2248-53.

6. Faden H, Duffy L, Wasielewski R, Wolf J, Krystofik $\mathrm{D}$, Tung $\mathrm{Y}$, et al. Relationship between nasopharyngeal colonization and the development of otitis media in children. J Infect Dis 1997;175:1440-5.

7. Homoe P, Prag J, Farholt S, Henrichsen J, Hornsleth A, Kilian M, et al. High rate of nasopharyngeal carriage of potencial pathogens among children in Greenland: results of a clinical survey of middle-ear disease. Clin Infect Dis 1996;23:1081-90.
8. Facklam RR, Washington JA. Streptococcus and related catalase-negative gram-positive cocci. In: Balows A, Hausler WJ Jr, Hermann KL, Isenberg HD, Shadomy $\mathrm{HJ}$, editors. Manual of Clinical Microbiology. 5th ed. Washington: American Society for Microbiology; 1991:. p.238-57.

9. Sorensen UBS. Typing of pneumococci by using 12 pooled antisera. J Clin Microbiol 1993;31:2097-100.

10. Nielsen SV, Henricksen J. Capsular types of Streptococcus pneumoniae isolated from blood and CSF during 1982-1987. Clin Infect Dis 1992;15:794-8.

11. Butler JC, Breiman RF, Lipman HB, Hofmann J, Facklam RR. Serotype distribution of Streptococcus pneumoniae infections among preschool children in the United States, 1978-1994: implications for development of a conjugate vaccine. J Infect Dis 1995;171:885-9.

12. Cifuentes M, Prado V, Pilorget JJ, Arrellano C, Prieto $\mathrm{S}$, Inostroza J, et al. Comparative prevalence of Streptococcus pneumoniae nasopharyngeal (NP) carriage among children and staff in Chilean day care centers (DCC). 9th International Congress on Infectious Diseases Abstracts. Buenos Aires (Argentina), April 1013, 2000. p.118.

13. O'Ryan M, Pilorget JJ, Mansor M, Prieto S, Arrellano $\mathrm{C}$, Inostroza J, et al. Longitudinal characterization of Streptococcus pneumoniae nasopharyngeal carriage during the 1998 winter-spring period in a Chilean DCC population. 9th International Congress on Infectious Diseases Abstracts. Buenos Aires (Argentina), April 1013, 2000. p.118. 